\title{
Models of the Short Interest Rate in Discrete Processes
}

\author{
Naoyuki Ishimura ${ }^{1}$, Bold Javkhlan ${ }^{1}$, MasaAki Nakamura ${ }^{2}$, Zheng Wei ${ }^{1}$ \\ ${ }^{1}$ Graduate School of Economics, Hitotsubashi University, Kunitachi,Tokyo 186-8601, Japan \\ ${ }^{2}$ College of Science and Technology, Nihon University, Kanda-Surugadai, Tokyo 101-8308, Japan \\ Email: ishimura@econ.hit-u.ac.jp,em112012@g.hit-u.ac.jp,nakamura@math.cst.nihon-u.ac.jp,em101011@g.hit-u.ac.jp
}

Received 2013

\begin{abstract}
The modeling of the term structure of interest rates is one of primary topics for researches in financial economics. Here we consider models of the short interest rate in discrete processes. Our methodology of analysis follows the framework of discrete stochastic calculus.
\end{abstract}

Keywords: Short Interest Rates; One-factor Model; Discrete Processes

\section{Introduction}

The term structure of interest rates has been the subject of intensive studies in financial economics. One major approach is to proclaim a priori the dynamics of the instantaneous (short) interest rate. For instance, in the context of one-factor model, the spot interest rate $r$ is assumed to follow a mean reverting process of the form

$$
r=\kappa(\beta-r) d t+\sigma r^{\beta} d W_{t},
$$

where $\beta \geq 0$ is a parameter, and $\kappa, \theta, \sigma$ are positive constants representing the rate ofreversion, long term interest rate, and the volatility of the interest rate, respectively. It is denoted by $d W_{t}$ the standard Brownian motion. After the famous work by Vasiček [1], the model (1) of the short interest rate has been extensively studied so far. We refer to, for instance, [1-7] and the references cited therein.

Here we are concerned with the model of the short interest rate in the case of discrete processes. A discrete version of Equation (1) is discussed; we mainly treat discrete Vasiček model, discrete Ho-Lee model, and discrete Hull-White model. Our methodology is based on the framework of discrete stochastic calculus [8-10]. The main goal of the current research is to compute the price of the discounted bond within these models, which will be presented in Section 4.

\section{Models}

Let $t=0,1,2, \cdots$ denote discrete time series and let $\left\{B_{t}\right\}_{t=0,1,2, \ldots}$ with $B_{0}=0$ be the one-dimensional random walk:

$$
B_{t}=\sum_{n=1}^{\mathrm{t}} Z_{n}
$$

where $\left\{Z_{n}\right\}_{n=1,2, \ldots}$ are independent and identically distributed (i.i.d.) random variables assumed to satisfy

$$
\mathrm{P}\left(Z_{n}=1\right)=\mathrm{P}\left(Z_{n}=-1\right)=\frac{1}{2}, n=1,2, \cdots
$$

That is, we treat the symmetric random walk. This restriction is just for simplicity and generalizations are also possible.

In this paper, the basic process $\left\{X_{t}\right\}_{t=0,1,2, \ldots}$ for the short rate is assumed to be given by the following discrete stochastic processes $([8,11])$.

$$
\begin{aligned}
& X_{t+1}-X_{t}=\mu\left(X_{t}, t\right)+\sigma\left(X_{t}, t\right)\left(B_{t+1}-B_{t}\right), \\
& t=0,1,2, \cdots,
\end{aligned}
$$

Where $\mu=\mu(x, t), \sigma=\sigma\left(X_{t}, t\right)$ are given continuous functions. The model corresponding to Equation (1) will be $r=r\left(X_{t}\right)$ with $\mu(x, t)=\kappa(\theta-x)$ and $\sigma(x, t)=\sigma x^{\beta}$.

The next examples of Equation (2) will be mainly considered in this article.

Example 1. Discrete Vasiček model ([1]) (discrete Ornstein-Uhlenbeck model).

$$
\mu(x, t)=a-b x, \sigma(x, t)=\sigma,
$$

where $a, b, \sigma$ are positive constants. We thus obtain

$$
X_{t+1}-X_{t}=a-b X_{t}+\sigma\left(B_{t+1}-B_{t}\right), \quad t=0,1,2, \cdots
$$

Example 2. Discrete Ho-Lee model ([5]).

$$
\mu(x, t)=\theta(t), \sigma(x, t)=\sigma,
$$

where $\theta=\theta(t)$ is a given function and $\sigma$ is a positive constant. We thus obtain

$$
X_{t+1}-X_{t}=\theta(t)+\sigma\left(B_{t+1}-B_{t}\right), t=0,1,2, \cdots .
$$

Example 3. Discrete Hull-White model ([6]) (discrete extended Vasiček model). 


$$
\mu(x, t)=\theta(t)-a(t) x, \sigma(x, t)=\sigma(t),
$$

where $\theta=\theta(t), a=a(t), \sigma=\sigma(t)$ are given functions. We thus obtain

$$
\begin{aligned}
& X_{t+1}-X_{t}=\theta(t)-a(t) X_{t}+\sigma(t)\left(B_{t+1}-B_{t}\right), \\
& t=0,1,2, \cdots
\end{aligned}
$$

\section{Basic Quantities}

Proposition 1. The expectation and the variance of each model are given as follows.

Example 1 (continued) (see [8]). It follows that

$$
\begin{aligned}
& \frac{X_{t+1}}{(1-b)^{t+1}}-\frac{X_{t}}{(1-b)^{t}}=\frac{a+\sigma Z_{t+1}}{(1-b)^{t+1}} \\
& X_{t}=(1-b)^{t} X_{0}-\frac{a}{b}\left((1-b)^{t}-1\right)+\sigma(1-b)^{t} \sum_{n=1}^{t} \frac{Z_{n}}{(1-b)^{n}},
\end{aligned}
$$

from which we have

$$
\begin{aligned}
E\left[X_{t}\right] & =(1-b)^{t} X_{0}-\frac{a}{b}\left((1-b)^{t}-1\right) \\
V\left[X_{t}\right] & =\sigma^{2} \frac{(1-b)^{2 t}-1}{(1-b)^{2}-1} .
\end{aligned}
$$

Example 2 (continued). It follows that

$$
X_{t}=X_{0}+\sum_{n=0}^{t-1} \theta_{n}+\sigma \sum_{n=1}^{t} Z_{n},
$$

from which we have

$$
E\left[X_{t}\right]=X_{0}+\sum_{n=0}^{t-1} \theta_{n}, \quad V\left[X_{t}\right]=\sigma^{2} t .
$$

Example 3 (continued). It follows that, for $t \geq 1$,

$$
\begin{aligned}
& X_{t}=\prod_{n=0}^{t-1}(1-a(n)) X_{0}+\sum_{n=1}^{t} \prod_{k=2}^{n}(1-a(t-k+1)) . \\
& \left(\theta(t-n)+\sigma(t-n) Z_{t+n-1}\right),
\end{aligned}
$$

from which we have

$$
\begin{aligned}
& E\left[X_{t}\right]=\sum_{n=1}^{t} \theta(t-n) \prod_{k=2}^{n}(1-a(t-k+1))+\prod_{n=0}^{t-1}(1-a(n)) X_{0}, \\
& V\left[X_{t}\right]=\sum_{n=1}^{t} \theta(t-n)^{2} \prod_{k=2}^{n}(1-a(t-k+1))^{2} .
\end{aligned}
$$

\section{Pricing Problems}

Here we compute the current price $P(0, T)$ of the discounted bond with maturity $T$ given in Examples of Section 2. In our setting, $P(0, T)$ is expressed as

$$
P(0, T)=E\left[\exp \exp \left(-\sum_{n=0}^{T} X_{n}\right)\right] .
$$

The results of computation are summarized in the next Theorem.
Theorem 2. The prices of the discounted bond of each model are computed as follows.

Example 1 (continued). Taking into account that $Z_{n}$ are independent, we deduce that

$$
\begin{array}{r}
P(0, T)=\exp \left(-\sum_{n=0}^{T}(1-b)^{n} X_{0}+\frac{a}{b}\left(1-(1-b)^{n}\right)\right) \\
\cdot E\left[\exp \left(-\frac{\sigma}{b} \sum_{n=1}^{T}\left(1-(1-b)^{n}\right) Z_{n}\right)\right] \\
=\exp \left(-\sum_{n=0}^{T}(1-b)^{n} X_{0}+\frac{a}{b}\left(1-(1-b)^{n}\right)\right) \\
\cdot \prod_{n=1}^{T} \cosh \cosh (\sigma(T-n+1)) .
\end{array}
$$

Example 2 (continued). We calculate

$$
\begin{aligned}
& P(0, T)=\exp \left(-\sum_{n=0}^{T} X_{0}-\sum_{n=0}^{T} \sum_{k=0}^{n-1} \theta_{k}\right) \cdot E\left[\exp \left(-\sigma \sum_{n=1}^{T} \sum_{k=1}^{n} Z_{k}\right)\right] \\
&=\exp \left(-(T+1) X_{0}-\sum_{n=0}^{T}(T-n) \theta_{n}\right) \\
& \cdot \prod_{n=1}^{T} \cosh \cosh (\sigma(T-n+1)) .
\end{aligned}
$$

Example 3 (continued). We compute

$$
\begin{aligned}
& P(0, T)=\exp \left(-\left(1+\sum_{n=1}^{T} \prod_{k=0}^{n-1}(1-a(k))\right) X_{0}\right) \\
& \cdot E\left[\exp \left(\begin{array}{c}
-\sum_{n=1}^{T} \sum_{k=1}^{n} \prod_{j=2}^{k}(1-a(n-j+1)) \\
\cdot\left(\theta(n-k)+\sigma(n-k) Z_{n+1-k}\right)
\end{array}\right)\right] \\
& =\exp \left(-\left(1+\sum_{n=1}^{T} \prod_{k=0}^{n-1}(1-a(k))\right) X_{0}\right) \\
& \cdot E\left[\exp \left(\begin{array}{c}
-\sum_{n=1}^{T} \sum_{k=1}^{n} \prod_{j=1}^{n-k-1}(1-a(j)) \\
\cdot\left(\theta(k)+\sigma(k) Z_{k+1}\right)
\end{array}\right)\right] \\
& =\exp \left(-\left(1+\sum_{n=1}^{T} \prod_{k=0}^{n-1}(1-a(k))\right) X_{0}\right) \\
& \cdot E\left[\exp \left(\begin{array}{c}
-\sum_{k=0}^{T-1} \sum_{n=k+1}^{T} \prod_{j=1}^{n-k-1}(1-a(j)) \\
\cdot\left(\theta(k)+\sigma(k) Z_{k+1}\right)
\end{array}\right)\right] \\
& =\exp \left(-\left(1+\sum_{n=1}^{T} \prod_{k=0}^{n-1}(1-a(k))\right) X_{0}\right) \\
& \cdot \exp \left(-\sum_{n=0}^{T-1} \sum_{k=n+1}^{T} \prod_{j=1}^{k-n-1}(1-a(j)) \theta(n)\right) \\
& \cdot \prod_{n=0}^{T-1} \cosh \left(\sum_{k=n+1}^{T} \prod_{j=1}^{k-n-1}(1-a(j)) \sigma(n)\right) .
\end{aligned}
$$




\section{Discussions}

We have dealt with instantaneous interest rate models indiscrete processes. An analogue of various well-known continuous short rate models is mainly investigated. The price of the discounted bond within these models is computed. The resulting formulas are somewhat complicated. The application in the real world, however, is rather expected. This is because of the readiness of calibration, which will be our next theme for researches.

\section{Acknowledgements}

The first author N. I. is supported in part by Grant-in-Aid for Scientific Research (A) No. 24243031, (C) No. 21540117 from Japan Society for the Promotion of Science (JSPS).

\section{REFERENCES}

[1] O. Vasiček, "An Equilibrium Characterization of the Term Structure," Journal of Financial Economics, Vol. 5, No. 2, 1977, pp. 177-188.

doi: 10.1016/0304-405X(77)90016-2

[2] T. Björk, "Arbitrage Theory in Continuous Time," 2nd edition., Oxford University Press, Oxford, 2004.

doi: 10.1093/0199271267.001.0001

[3] J. Cox, J. Ingersoll and S. Ross, “A Theory of the Te
Structure of Interest Rate," Econometrica, Vol. 53, No. 2, 1985 , pp. $385-408$.

doi: $10.2307 / 1911242$

[4] D. Heath, R. Jarrow and A. Morton, "Bond Pricing and the Term Structure of Interest Rates," Econometrica, Vol. 60, No. 1, 1992, pp. 77-106. doi: 10.2307/2951677

[5] T. Ho and S. Lee, "Term Structure Movements and Pricing Interest Rate Contingent Claims," The Journal of Finance, Vol. 41, No. 5, 1986, pp. 1011-1029.

doi: 10.1111/j.1540-6261.1986.tb02528.x

[6] J. Hull and A. White, "Pricing Interest-rate-derivative Securities," Review Financial Studies, Vol. 3, No. 4, 1990, pp. 573-592. doi: $10.1093 / \mathrm{rfs} / 3.4 .573$

[7] A. Pelsser, "Efficient Methods for Valuing Interest Rate Derivatives," Springer, London, 2000.

doi: 10.1007/978-1-4471-3888-4

[8] T. Fujita, "Introduction to the Stochastic Analysis for Financial Derivatives (Finance No Kakuritsu-Kaiseki Nyumon)," Kodan-shya, Tokyo, Japanese. 2002

[9] T. Fujita, N. Ishimura and N. Kawai, "Discrete Stochastic Calculus and Its Applications: An Expository Note," Advances in Mathematics Economics, Vol. 16, 2012, pp. 119-131. doi: 10.1007/978-4-431-54114-1 6

[10] T. Fujita and Y. Kawanishi, "A Proof of Itº's Formula Using a Discrete Itô's Formula," Stud. Scienti. Math. Hungarica, Vol. 45, 2008, pp. 125-134.

[11] A. V. Mel'nikov, "Financial Markets," American Mathematical Society, Providence, 1999. 